\title{
Structural and Functional Changes of Myocardium Under Conditions of Postinfarcate Cardiosclerosis, Arterial Hypertension, Type 2 Diabetes Mellitus
}

\section{NO Kravchun ${ }^{1 *}$, IP Dunaieva ${ }^{2}$, IA Ilchenko² ${ }^{2}$ PP Kravchun ${ }^{3}$ and IV Cherniavskaia ${ }^{4}$}

${ }^{1}$ Multidisciplinary Medical Center "Life Park", Kharkiv, Ukraine

${ }^{2}$ Kharkiv National Medical University of the Ministry of Health of Ukraine, Kharkiv, Ukraine

${ }^{3} I Q V I A$ RDS, Kyiv, Ukraine

${ }^{4}$ Medical Center "REFIT", Kharkiv, Ukraine

*Corresponding Author: NO Kravchun, Multidisciplinary Medical Center "Life

Park", Kharkiv, Ukraine.
Received: October 07, 2021

Published: November 23, 2021

(C) All rights are reserved by NO Kravchun., et al.

\begin{abstract}
The article evaluates the structural and functional changes of the myocardium in patients with postinfarction cardiosclerosis, hypertension and type 2 diabetes depending on the systolic function of the left ventricle and the functional class of chronic heart failure. A study of 68 patients with postinfarction cardiosclerosis, hypertension and type 2 diabetes mellitus was performed. The comparison group included 60 patients with postinfarction cardiosclerosis, the control group - 30 almost healthy individuals. Type 2 diabetes mellitus is a potentiator of left ventricular myocardial remodeling in patients with systolic dysfunction caused by postinfarction cardiosclerosis. Comorbidity of postinfarction cardiosclerosis, arterial hypertension and type 2 diabetes mellitus leads to the progression of postinfarction remodeling.
\end{abstract}

Keywords: Postinfarction Cardiosclerosis; Type 2 Diabetes Mellitus; Chronic Heart Failure; Systolic Dysfunction

\section{Introduction}

Comorbidity is nowadays one of the most important problems of modern medicine. Given that the cardiovascular diseases (CVD) are in the lead in the mortality structure, the scientists' attention is riveted on early diagnostics of CVD, improvement of the effectiveness of primary and secondary prevention.

Ischemic heart disease (IHD), arterial hypertension (AH) and diabetes mellitus (DM) are the common diseases and their prevalence rate is constantly increasing [1].

AH prevalence in patients with 2 type DM is up to three times higher than in patients without DM, and their combination significantly increases the likelihood of CVD development [2-7]. The unfavorable interrelation between these two conditions can cause negative pathophysiological changes in the cardiovascular system, as it significantly accelerates the atherosclerosis processes. AH and 2 type DM have several common pathophysiological mechanisms, that justify the fact of their comorbidity. Insulin resistance (IR), hyperinsulinemia, oxidative stress (OS) activation and subclinical chronic systemic low-intensity inflammation are the main best studied pathophysiological components $[5,8]$.

The peculiarities of systolic and diastolic dysfunction in patients with IHD, after previous myocardial infarction (MI), caused by structural and functional changes of myocardium, geometry of 
the left ventricle (LV), its remodeling, are currently considered depending on the presence of complex of comorbide conditions and diseases, which, in the first instance include AH, 2 type DM [9-12].

The data collected on the role of structural and functional changes of myocardium in CVD pathogenesis due to the development and progression of chronic heart failure (CHF) resulted in the formation of concept about the indicators geometry of the left ventricle, as a target for prevention and treatment of pathological processes, which implement CVD by means of LV remodeling, especially in patients after previous IM [13-16].

These provisions have substantiated a new trend of fundamental and clinical research, namely studying the mechanisms of participation of myocardium morpho-functional changes in the pathogenesis and development of systolic dysfunction and CHF in patients with IHD, AH and 2 type DM, as well as the methods of effective therapy. Especially since the very control of IHD clinical manifestations associated with DM, without correction of myocardial remodeling, cannot be considered a successful clinical case.

The innovative trend is the study of structural and functional parameters of myocardium and the impact on these comorbide pathology indicators, namely IHD, $\mathrm{AH}$ and 2 type DM, which aggravating effect is caused by the pathogenesis unity.

The study objective is the assessment of LV geometry parameters in patients with postinfarction cardiosclerosis, $\mathrm{AH}$ and coexisting 2 type DM depending on the LV systolic function and functional class (FC) of chronic heart failure (CHF).

In retrospect, the study included 68 patients with postinfarction cardiosclerosis, $\mathrm{AH}$ and coexisting 2 type DM. The mean age of the persons under examination was $(64.38 \pm 1.08)$ years, including 32 men $(47.0 \%)$ and 36 women (53.0\%). The study did not involve patients with severe comorbidities of the respiratory, digestive, renal system and cancer. The experimental group was composed of 60 patients with postinfarction cardiosclerosis without diabetes, with the mean age of $(63.47 \pm 1.28)$ years -32 men $(53.3 \%)$ and 28 women (46.7\%). The groups were comparable in age and gender. The control group included 30 almost healthy persons; their mean age was $(58.23 \pm 1.36)$ years.

$\mathrm{AH}$ diagnosis, determination of $\mathrm{AH}$ stage was carried out according to the latest recommendations of the European Society of Cardiology (ESC), European Society of Hypertension (ESH) and International Society of Hypertension (ISH) [17-19].

All patients underwent general clinical and instrumental examinations. Echocardiographic examinations were carried out by standard method of Kh. Feigenbaum using RADMIR ultrasound equipment (Ultima PRO 30) (Kharkiv, Ukraine). The following LV parameters were determined in M-mode: end-diastolic dimension (EDD) (cm), end-systolic dimension (ESD) (cm), posterior wall thickness (LVPWT) (cm), interventricular septum thickness (IVST) (cm). LV end-diastolic volume (EDV) and systolic volume (ESV) (ml) were calculated by Simpson method (1991), then LV ejection fraction (EF) was computed (\%). LV mass (LVM) was calculated by the formula of R. Devereux., et al. $1.04 \times$ [(IVST+LVPWT+EDD)3] [EDD]3 - 13.6. LV myocardium wall thickness index (LVMWTI) was calculated by the formula: LVMWTI=(LVPWTd+IVSTd)/EDD. LV mass index (LVMI) was calculated in relation to a patient's growth: LVMI $\left(\mathrm{g} / \mathrm{m}^{2}\right)=\mathrm{LVM} / \mathrm{BSA}$, where BSA is body surface area $\left(\mathrm{m}^{2}\right)$. Also, the left atrium (LA) $(\mathrm{cm})$ was determined by the dimension between LA posterior wall and posterior aortic wall in the parasternal image along the long axis. The aorta $(\mathrm{cm})$ was measured in the parasternal image along the long axis to the aortic axis in four places from the anterior border of proximal wall to the anterior border of distal wall at the end of systole and at the end of diastole. The aortic dimensions are indexed to the square root of the patient's body surface area. For more accurate assessment of the geometric type of LV remodeling, relative posterior wall thickness (LVRPWT) and relative interventricular septum thickness (RIVST) were determined separately, which allows to identify the asymmetric nature of LV geometry by the formulas: LVRPWT $=(2 \times$ LVPWT $) /$ EDD and RIVST $=(2 \times I V S T) /$ EDD.

Statistical processing of the data obtained was carried out using the statistical software package Statistica 8,0 (StatSoft Inc, USA), Microsoft Office Excel-2003. The quantitative characters at normal distribution were submitted in the form of the mean \pm standard error of the mean $(\mathrm{M} \pm \mathrm{m})$; in order to compare the means of two samples, Student's t-test was used. For all types of test, the differences were considered statistically significant at $\mathrm{p}<0.05$.

Study results and their discussion

The comparison of cardiac hemodynamic indicators in the groups comparing patients with postinfarction cardiosclerosis and AH depending on the presence of 2 type DM showed the meaningful 
changes of the following indicators: LVPWT, IVST, RIVST, LVRPWT, LVMI and LVMWTI ( $<0.05)$. Thus, LVPWT was greater by8.0\%, IVST - by $9.5 \%$, RIVST - by $24.0 \%$, LVRPWT - by $30.0 \%$, LVMI - by $11.0 \%$, and LVMWTI - by $15.0 \%(\mathrm{p}<0.05)$ in patients in case of the combination of postinfarction cardiosclerosis, AH and 2 type DM. Meanwhile, the mean values of indicators LVPWT, IVST, RIVST, LVRPWT, LVMI and LVMWTI exceeded their values in the experimental group, which is illustrative of the presence of LV hypertrophy in patients, particularly its concentric type, as well as it can be caused by the protracted $\mathrm{AH}$.

Therefore, it is proved that 2 type DM is a predictor of LV concentric hypertrophy development in patients with postinfarction cardiosclerosis, which is a response of the heart to the prolonged load increase and microcirculatory disorders in myocardium; it also results in the complex structural and functional reorganization of the heart with mostly concentric remodeling and LV concentric hypertrophy with a tendency to decrease myocardial contractility.

In order to determine the specific effect of 2 type DM on the structural and functional state of the left ventricle, the study groups were compared depending on EF. Table 1 shows the data of the examined patients depending on EF.

\begin{tabular}{|l|c|c|c|}
\hline Indicator & $\begin{array}{c}\mathbf{E F}>\mathbf{4 5 . 0} \\
(\mathbf{n}=\mathbf{3 5})\end{array}$ & $\begin{array}{c}\mathbf{E F}<\mathbf{4 5 . 0} \mathbf{( \mathbf { 0 }} \mathbf{3 5}) \\
\mathbf{( n )}\end{array}$ & \\
\hline Age, year & $62.45 \pm 1.02$ & $65.61 \pm 1.03$ & $>0.05$ \\
\hline ESD, cm & $3.18 \pm 0.10$ & $4.51 \pm 0.08$ & $<0.05$ \\
\hline EDD, cm & $5.26 \pm 0.10$ & $7.54 \pm 0.11$ & $<0.05$ \\
\hline LVPWT, cm & $1.47 \pm 0.02$ & $1.49 \pm 0.03$ & $>0.05$ \\
\hline IVST, c, & $1.45 \pm 0.03$ & $1.47 \pm 0.02$ & $>0.05$ \\
\hline LVMI, g/m ${ }^{2}$ & $159.30 \pm 4.32$ & $135.54 \pm 4.51$ & $<0.05$ \\
\hline EDV, ml & $150.50 \pm 3.46$ & $185.34 \pm 3.73$ & $<0.05$ \\
\hline ESV, ml & $76.66 \pm 1.90$ & $98.90 \pm 2.10$ & $<0.05$ \\
\hline LVM, g & $243.22 \pm 6.80$ & $295.57 \pm 7.20$ & $<0.05$ \\
\hline LA, cm & $3.56 \pm 0.06$ & $5.26 \pm 0.08$ & $<0.05$ \\
\hline Aorta, cm & $2.76 \pm 0.04$ & $3.84 \pm 0.04$ & $<0.05$ \\
\hline
\end{tabular}

Table 1: Characteristics of the structural and functional state of the left ventricle in patients with postinfarction cardiosclerosis, $\mathrm{AH}$ and 2 type $\mathrm{DM}, \mathrm{M} \pm \mathrm{m}$.
As expected, the patients with reduced EF ( $\leq 45.0 \%)$ compared with patients with preserved EF ( $>45.0 \%)$ showed records of the probable increase in ESD by 29.49\% (p < 0.05), EDD - by $30.24 \%$ (p $<0.05$ ), LVMI - by 14.91\% (p < 0.05), EDV - by 18.80\% ( $<<0.05)$, ESV - by $22.49 \%$ ( $<<0.05)$, LVM - by $17.71 \%$ ( $<<0.05)$, LA dimensions - by $32.32 \%(\mathrm{p}<0.05)$ and aorta diameter- by $28.13 \%$ ( $<<$ $0.05)$. At the same time, no significant differences were found between the groups in the ratio of IVST and LVPWT ( $p>0.05$ ). These results allow making a conclusion that patients with postinfarction cardiosclerosis, $\mathrm{AH}$ and 2 type DM with reduced EF have more profound disorders of LV myocardium structure and function.

Table 2 shows structural and functional parameters of LV in patients with postinfarction cardiosclerosis, $\mathrm{AH}$ and coexisting 2 type DM depending on CHF FC.

\begin{tabular}{|c|c|c|c|c|}
\hline \multirow[t]{2}{*}{ Indicator } & \multicolumn{3}{|c|}{ Group, number of patients } & \multirow[t]{2}{*}{$\mathbf{P}$} \\
\hline & $\begin{array}{c}\text { II FC CHF, } \\
n=22\end{array}$ & $\begin{array}{c}\text { III FC CHF, } \\
n=29\end{array}$ & $\begin{array}{c}\text { IV FC CHF, } \\
n=17\end{array}$ & \\
\hline EDV, ml & $\begin{array}{c}149.46 \pm \\
2.53\end{array}$ & $\begin{array}{c}163.36 \pm \\
2.33\end{array}$ & $\begin{array}{c}189.45 \pm \\
2.76\end{array}$ & $\begin{array}{l}\mathrm{p}_{1-2}<0.05 \\
\mathrm{p}_{1-3}<0.05 \\
\mathrm{p}_{2-3}<0.05\end{array}$ \\
\hline $\mathrm{ESV}, \mathrm{ml}$ & $\begin{array}{c}75.81 \pm \\
1.71\end{array}$ & $82.16 \pm 1.52$ & $\begin{array}{c}105.37 \pm \\
1.46\end{array}$ & $\begin{array}{l}\mathrm{p}_{1-2}<0.05 \\
\mathrm{p}_{1-3}<0.05 \\
\mathrm{p}_{2-3}<0.05\end{array}$ \\
\hline EDD, $\mathrm{cm}$ & $\begin{array}{c}5.54 \pm \\
0.09\end{array}$ & $6.11 \pm 0.04$ & $7.52 \pm 0.07$ & $\begin{array}{l}\mathrm{p}_{1-2}<0.05 \\
\mathrm{p}_{1-3}<0.05 \\
\mathrm{p}_{2-3}<0.05\end{array}$ \\
\hline $\mathrm{ESD}, \mathrm{cm}$ & $\begin{array}{c}3.12 \pm \\
0.04\end{array}$ & $3.76 \pm 0.08$ & $4.65 \pm 0.05$ & $\begin{array}{l}\mathrm{p}_{1-2}<0.05 \\
\mathrm{p}_{1-3}<0.05 \\
\mathrm{p}_{2-3}<0.05\end{array}$ \\
\hline $\mathrm{EF}, \%$ & $\begin{array}{c}51.57 \pm \\
0.91\end{array}$ & $49.64 \pm 1.23$ & $\begin{array}{c}43.18 \pm \\
1.23\end{array}$ & $\begin{array}{l}\mathrm{p}_{1-2}>0.05 \\
\mathrm{p}_{1-3}<0.05 \\
\mathrm{p}_{2-3}<0.05\end{array}$ \\
\hline LVPWT, cm & $\begin{array}{c}1.48 \pm \\
0.04\end{array}$ & $1.50 \pm 0.02$ & $1.47 \pm 0.05$ & $\begin{array}{l}\mathrm{p}_{1-2}>0.05 \\
\mathrm{p}_{1-3}>0.05 \\
\mathrm{p}_{2-3}>0.05\end{array}$ \\
\hline IVST, cm & $\begin{array}{c}1.43 \pm \\
0.02\end{array}$ & $1.49 \pm 0.04$ & $1.46 \pm 0.01$ & $\begin{array}{l}\mathrm{p}_{1-2}>0.05 \\
\mathrm{p}_{1-3}>0.05 \\
\mathrm{p}_{2-3}>0.05\end{array}$ \\
\hline
\end{tabular}




\begin{tabular}{|c|c|c|c|c|}
\hline LA, cm, & $3.96 \pm$ & $4.28 \pm 0.09$ & $5.01 \pm 0.11$ & $\mathrm{p}_{1-2}<0.05$ \\
& 0.12 & & & $\begin{array}{c}\mathrm{p}_{1-3}<0.05 \\
\mathrm{p}_{2-3}<0.05\end{array}$ \\
\hline Aorta, cm & $\begin{array}{c}3.28 \pm \\
0.04\end{array}$ & $3.30 \pm 0.06$ & $3.33 \pm 0.03$ & $\mathrm{p}_{1-2}>0.05$ \\
& & & & $\begin{array}{l}\mathrm{p}_{1-3}>0.05 \\
\mathrm{p}_{2-3}>0.05\end{array}$ \\
\hline LVM, g & $234.32 \pm$ & $275.44 \pm$ & $298.51 \pm$ & $\mathrm{p}_{1-2}<0.05$ \\
& 5.70 & 6.30 & 5.20 & $\mathrm{p}_{1-3}<0.05$ \\
& & & & $\mathrm{p}_{2-3}<0.05$ \\
\hline LVMWTI & $0.52 \pm$ & $0.51 \pm 0.06$ & $0.54 \pm 0.01$ & $\mathrm{p}_{1-2}>0.05$ \\
& 0.08 & & & $\mathrm{p}_{1-3}>0.05$ \\
& & & & $\mathrm{p}_{2-3}>0.05$ \\
\hline LVMI, g/m ${ }^{2}$ & $128.53 \pm$ & $138.76 \pm$ & $173.97 \pm$ & $\mathrm{p}_{1-2}<0.05$ \\
& 5.09 & 4.46 & 6.11 & $\mathrm{p}_{1-3}<0.05$ \\
& & & & $\mathrm{p}_{2-3}<0.05$ \\
\hline
\end{tabular}

Table 2: Structural and functional parameters of LV in patients with postinfarction cardiosclerosis, $\mathrm{AH}$ and coexisting 2 type $\mathrm{DM}$ depending on $\mathrm{CHF} F \mathrm{FC}, \mathrm{M} \pm \mathrm{m}$.

Analysis of the study results of central hemodynamics in patients with postinfarction cardiosclerosis, $\mathrm{AH}$ and coexisting 2 type DM depending on CHF FC showed that with CHF progression there was a probable increase in EDV, ESV, EDD, ESD levels, LA, LVM and LVMI dimensions, as well reduction of EF ( $p<0.05)$. As for such indicators as LVPWT, IVST, aorta dimension and LVMWTI, no significant differences were found depending on CHF FC ( $p>0.05$ ). Thus, the patients with CHF IV FC developed associated with postinfarction cardiosclerosis, AH and 2 type DM have EDV greater by $13.77 \%$ and $21.11 \%$, ESV - by $22.03 \%$ and $28.05 \%$, EDD - by $18.75 \%$ and $26.33 \%$, ESD - by $19.14 \%$ and $32.9 \%$, LA dimension- by $14.57 \%$ and $20.96 \%$, LVM - by $7.73 \%$ and $21.5 \%$, LVMI - by $20.24 \%$ and $26.12 \%$ compared to the patients with III FC CHF and II FC CHF correspondingly ( $\mathrm{p}<0.05)$. EF, in contrast, tended to decrease in patients with II FC CHF compared to patients with III FC CHF, but no significant differences were found ( $p>0.05$ ). Whereas the patients with IV FC CHF have EF significantly lower by $13.01 \%$ and $16.27 \%$, than patients with III FC CHF and II FC CHF correspondingly $(\mathrm{p}<0.05)$.

Therefore, the increase in CHF FC in patients with postinfarction cardiosclerosis was associated with the progression of LV hy- pertrophy, decreased inotropic myocardial function, increased dimension and volume of LV cavity, deterioration of functional status, which is undoubtedly a consequence of the underlying disease and 2 type DM.

The results we obtained do not contradict with the results of other researchers [20-23] and make it possible to state that the mechanisms underlying the disease are associated with impaired glucose and insulin metabolism, leading to LV remodeling in patients with postinfarction cardiosclerosis, $\mathrm{AH}$ and 2 type DM.

\section{Conclusion}

- $\quad$ Type 2 diabetes mellitus is a potentiator of left ventricular myocardial remodeling in patients with systolic dysfunction caused by postinfarction cardiosclerosis.

- Comorbidity of postinfarction cardiosclerosis, arterial hypertension and type 2 diabetes mellitus leads to the increasing difficulty of postinfarction remodeling associated with increase in the functional class of chronic heart failure.

\section{Bibliography}

1. V Ormazabal., et al. "Association between insulin resistance and the development of cardiovascular disease". Cardiovascular Diabetology 17 (2018): 122.

2. Petrie JR., et al. "Diabetes, Hypertension, and cardiovascular disease: Clinical Insights and Vascular Mechanisms". Canadian Journal of Cardiology 34.5 (2018): 575-584.

3. Zhou MS., et al. "Link between the renin-angiotensin system and insulin resistance: implications for cardiovascular disease". Vascular Medicine 17.5 (2012): 330-341.

4. B Williams., et al. "ESC/ESH Guidelines for the management of arterial hypertension". European Heart Journal 39.33 (2018): 3021-3104.

5. Serhiyenko V and Serhiyenko O. "Diabetes mellitus and arterial hypertension". International journal of endocrinology (Ukraine) 17.2 (2021): 175-188.

6. G Lastra., et al. "Type 2 diabetes mellitus and hypertension: an update". Endocrinology and Metabolism Clinics of North America 43.1 (2014): 103-122. 
7. Savoia C and Touyz RM. "Hypertension, Diabetes Mellitus, and Excess Cardiovascular Risk: Importance of Baseline Systolic Blood Pressure". Hypertension 70.5 (2017): 882-883.

8. Gorbunova 0., et al. "The level of protection from oxidative stress and three-year dynamics of structural and functional changes of the myocardium in chronic ischemic heart disease in men". Georgian Medical News 285 (2018): 63-69.

9. M Fudim., et al. "The Prognostic Value of Diastolic and Systolic Mechanical Left Ventricular Dyssynchrony Among Patients with Coronary Heart Disease". JACC Cardiovascular Imaging 2.7 (2019): 1215-1226.

10. Y Lin., et al. "Effect of Trimetazidine on Diabetic Patients with Coronary Heart Diseases: A Meta-Analysis of Randomized, Controlled Trials". Chinese Medical Sciences Journal 35.3 (2020): 226-238.

11. Chen Y., et al. "Blood glucose fluctuations in patients with coronary heart disease and diabetes mellitus correlates with heart rate variability: A retrospective analysis of 210 cases". Nigerian Journal of Clinical Practice 23.9 (2020): 1194-1200.

12. SJ Shah., et al. "Prevalence and correlates of coronary microvascular dysfunction in heart failure with preserved ejection fraction: PROMIS-HFpEF". European Heart Journal 39.37 (2018): 3439-3450.

13. Zhou D., et al. "Uncontrolled hypertension increases risk of all-cause and cardiovascular disease mortality in US adults: the NHANES III Linked Mortality Study". Scientific Reports 8.1 (2018): 9418.

14. Frangogiannis NG and Kovacic JC. "Extracellular Matrix in Ischemic Heart Disease, Part 4/4: JACC Focus Seminar". Journal of the American College of Cardiology 75.17 (2020): 2219-2235.

15. Verbovoy AF., et al. "Diabetic macroangiopathy". Therapeutic archive 91.10 (2019): 139-143.

16. AF Verbovoy., et al. "Modern approaches to management of cardiovascular risk factors at patients with diabetes mellitus type 2". Therapeutic archive 90.8 (2018): 113-117.

17. NR Poulter., et al. "Are the American Heart Association/American College of Cardiology High Blood Pressure Guidelines Fit for Global Purpose? Thoughts From the International Society of Hypertension". Hypertension 72.2 (2018): 260-262.
18. B Williams., et al. "2018 ESC/ESH Guidelines for the management of arterial hypertension: The Task Force for the management of arterial hypertension of the European Society of Cardiology and the European Society of Hypertension: The Task Force for the management of arterial hypertension of the European Society of Cardiology and the European Society of Hypertension". Journal of Hypertension 39.33 (2018): 30213104.

19. T Unger., et al. "2020 International Society of Hypertension Global Hypertension Practice Guidelines". Hypertension 75.6 (2020): 1334-1357.

20. Green JB. "Understanding the type 2 diabetes mellitus and cardiovascular disease risk paradox". Postgraduate Medical 126.3 (2014): 190-204.

21. M Guglin., et al. "Heart failure as a risk factor for diabetes mellitus". Cardiology 129.2 (2014): 84-92.

22. H Ohuchi., et al. "Low fasting plasma glucose level predicts morbidity and mortality in symptomatic adults with congenital heart disease". International Journal of Cardiology 174.2 (2014): 306-312.

23. C Varas-Lorenzo., et al. "The risk of heart failure associated with the use of noninsulin blood glucose-lowering drugs: systematic review and metaanalysis of published observational studies". BMC Cardiovascular Disorders 14.1 (2014): 129.

\section{Volume 4 Issue 12 December 2021 (C) All rights are reserved by NO Kravchun., et al.}

